\title{
Formation and Characterization of Copper Nanocube-Decorated Reduced Graphene Oxide Film
}

\author{
M. Z. H. Khan, ${ }^{1}$ M. A. Rahman, ${ }^{1}$ P. Yasmin, ${ }^{1}$ F. K. Tareq, ${ }^{1}$ N. Yuta, ${ }^{2}$ \\ T. Komeda, ${ }^{3}$ and R. A. Jahan ${ }^{4}$ \\ ${ }^{1}$ Department of Chemical Engineering, Jessore University of Science and Technology, Jessore 7408, Bangladesh \\ ${ }^{2}$ Research Core for Interdisciplinary Sciences, Okayama University, Tsushimanaka, Kita-ku, Okayama 700-8530, Japan \\ ${ }^{3}$ Institute of Multidisciplinary Research for Advanced Materials (IMRAM), Tohoku University, 2-1-1, Katahira, Aoba-Ku, \\ Sendai 980-0877, Japan \\ ${ }^{4}$ Center for Advanced Research in Sciences, University of Dhaka, Dhaka 1000, Bangladesh
}

Correspondence should be addressed to M. Z. H. Khan; zaved.khan@yahoo.com

Received 31 January 2017; Revised 13 April 2017; Accepted 8 May 2017; Published 4 June 2017

Academic Editor: Giuseppe Compagnini

Copyright (C) 2017 M. Z. H. Khan et al. This is an open access article distributed under the Creative Commons Attribution License, which permits unrestricted use, distribution, and reproduction in any medium, provided the original work is properly cited.

In this study, we present a new approach for the formation and deposition of $\mathrm{Cu}$ nanocube-decorated reduced graphene oxide (rGO-CuNCs) nanosheet on indium tin oxide (ITO) electrode using very simple method. Cubic Cu nanocrystals have been successfully fabricated on rGO by a chemical reduction method at low temperature. The morphologies of the synthesized materials were characterized by ultraviolet-visible (UV-vis) spectroscopy, scanning electron microscopy (SEM), Fourier transform infrared (FTIR) spectroscopy, and atomic force microscopy (AFM). The as-formed CuNCs were found to be homogeneously and uniformly decorated on rGO nanosheets. We demonstrated that the individual rGO sheets can be readily reduced and decorated with CuNCs under a mild condition using L-ascorbic acid (L-AA). Such novel ITO/rGO-CuNCs represent promising platform for future device fabrication and electrocatalytic applications.

\section{Introduction}

In recent years, graphene oxide (GO) and reduced graphene oxide ( $\mathrm{rGO}$ ) became a hot topic and opened an entirely new domain in the area of material science [1-3]. The opportunity to combine GO with inorganic nanoparticles, thereby forming graphene-nanoparticle hybrid structures, is currently of interest for applications in bioelectronics, energy production, biomedicine, and environmental remediation [2-7]. These graphene-nanoparticle hybrid structures exhibit additional advantageous and often synergistic properties that greatly augment their potential for bioapplications. For instance, the decoration of metal or metal oxide nanoparticles on graphene surfaces has been shown to have implications on the charge transfer behavior in graphene through the modification of the local electronic structure.

The combination of GO with inorganic nanoparticles has led to nanocomposites with multiple functionalities that are protected against degradation by coating with a suitable material (shell) $[8,9]$ and currently of interest for applications in biomedicine, energy production, electronics, and environmental remediation. In previous several researchers reported about $\mathrm{rGO}-\mathrm{Cu}$ core-shell nanostructures synthesized by pyrolysis of organocopper [10], CVD method [11], and hydrothermal process [12].

Previously, Wang et al. have reported reduction/deposition of reduced graphene oxide/copper nanoparticles (rGO/ $\mathrm{Cu}$ NPs) on electrode surface for biosensing application [13]. In another work, Xiong et al. have fabricated bulk rGO$\mathrm{Cu}$ nanolaminated composite by bottom-up assembly and reported the high reinforcing efficiency of graphene in metal matrix [14]. It was established that copper hydroxide/oxide layer in situ formed on $\mathrm{Cu}$ nanocube surfaces plays an important role in enhancing the electrocatalytic activity and stability of the hydrazine electrocatalyst [15]. Other researchers successfully fabricated $\mathrm{Cu}_{2} \mathrm{O}$ nanocubes wrapped by graphene nanosheets $\left(\mathrm{Cu}_{2} \mathrm{O} / \mathrm{GNs}\right)$ that represent promising 
enzyme-free glucose and hydrogen peroxide sensors with high sensitivity and selectivity [16].

Herein, we present the fabrication of copper nanocubes(CuNCs-) decorated reduced graphene oxide (rGO) nanocomposite. The nanocomposite was prepared using simple chemical reduction method, consisting of simultaneous reduction of GO and formation of copper nanocubes with Lascorbic acid at room temperature.

\section{Materials and Methods}

2.1. Chemicals and Instruments. All chemicals were reagent grade or higher and were used as received unless otherwise specified. ITO electrode $\left((1500 \pm 200 \AA) / \mathrm{SiO}_{2}(250 \AA) /\right.$ glass $(0.7 \mathrm{t} \times 10 \mathrm{~mm} \times 10 \mathrm{~mm})$, with a sheet resistance of $\leq 15 \mathrm{ohm} / \mathrm{sq}$ ) was purchased from Sanyo Vacuum Industry, Japan. Graphite powder, potassium permanganate $\left(\mathrm{KMnO}_{4}\right)$, sulphuric acid $\left(\mathrm{H}_{2} \mathrm{SO}_{4}\right)$, phosphoric acid $\left(\mathrm{H}_{3} \mathrm{PO}_{4}\right)$, hydrogen peroxide $\left(\mathrm{H}_{2} \mathrm{O}_{2}\right)$, copper sulphate $\left(\mathrm{CuSO}_{4}\right)$, sodium hydroxide $(\mathrm{NaOH})$, glucose, uric acid (UA), and L-ascorbic acid (AA) were purchased from Sigma-Aldrich. Ultrapure water was used throughout the experiments. The morphology and structure of GO were characterized by AFM (PSIA XE-100, Park System) and field-emission scanning electron microscopy (SEM, JSM-6700F). SEM is equipped to perform elemental chemical analysis by energy dispersive X-ray spectroscopy (EDS). Fourier transform infrared (FTIR) method was employed to characterize the nanocomposite, and IR spectra were recorded on Perkin-Elmer FTIR-1710 spectrophotometer using $\mathrm{KBr}$.

2.2. Preparation of Graphene Oxide (GO). The GO sheets used in this study were synthesized from purified natural graphite (SP-1, Bay Carbon) by modified Hummers method using $300 \mathrm{wt} \%$ of $\mathrm{KMnO}_{4}$ for graphite [17]. Graphite (3.0 g) was stirred in $95 \% \mathrm{H}_{2} \mathrm{SO}_{4}(75 \mathrm{~mL})$. The required amount of $\mathrm{KMnO}_{4}(6.0$ and $15 \mathrm{~g})$ was gradually added to the solution keeping the temperature $<10^{\circ} \mathrm{C}$. The mixture was then stirred at $35^{\circ} \mathrm{C}$ for $2 \mathrm{~h}$. The resulting mixture was diluted by water $(75 \mathrm{~mL})$ under vigorous stirring and cooling so that temperature does not exceed $50^{\circ} \mathrm{C}$. The suspension was further treated by adding $30 \%$ aq. $\mathrm{H}_{2} \mathrm{O}_{2}(7.5 \mathrm{~mL})$. The resulting graphite oxide suspension was purified by centrifugation with water until neutralization. Several GOs were analyzed by CHNS elemental analysis to evaluate the oxygen content.

2.3. Preparation of $r G O-C u N C s$. At first GO solution was dispersed in deionized water and sonicated for $1 \mathrm{hr}$ after vigorous stirring for $30 \mathrm{~min}$. Then preprepared saturated solutions of copper sulfate $(1 \mathrm{ml})$ and $\mathrm{L}$-ascorbic acid $(3 \mathrm{ml})$ were gradually added into aqueous dispersion of the GO. The reduction was performed at $90^{\circ} \mathrm{C}$ for $4 \mathrm{hrs}$ with continuous stirring. After completing reaction, rGO-CuNCs nanocomposites were obtained as precipitation and the solution color changed into dark brown. The resultant precipitates were simply centrifuged and filtered by cellulose filter paper and further were washed with a $1 \mathrm{M}$ hydrochloric acid solution (37\%, Merck) and distilled water to neutral $\mathrm{pH}$. The final nanocomposites were dried at $80^{\circ} \mathrm{C}$. Finally, the filtrate was dried at $90^{\circ} \mathrm{C}$ to obtain $\mathrm{rGO}-\mathrm{CuNCs}$ nanocomposites powder.

2.4. Preparation of ITO/rGO-CuNCs. The rGO-CuNCs nanocomposites were deposited on ITO electrode by dipping method. At first the rGO-CuNCs nanocomposites were dispersed in deionized water and sonicated for $1 \mathrm{hr}$. Then the cleaned ITO electrodes were immersed in aqueous solution of rGO-CuNCs for 12 hours at room temperature. After rinsing with pure water several times and being dried, the modified ITO/rGO-CuNCs electrodes were kept in vacuum place for characterization.

\section{Results and Discussion}

After reduction of the GO by L-AA, the color of the GO suspension changed from light brown to dark brown (Scheme 1). The dark color of the rGO indicates removal of oxygencontaining bonds, resulting in electronic conjugation within reduced sheets. UV-vis spectroscopy was used to monitor the reduction progress. As shown in Figure 1(a), GO exhibited a maximum absorption peak at approximately $235 \mathrm{~nm}\left(\pi-\pi^{*}\right.$ transitions of aromatic $\mathrm{C}=\mathrm{C}$ bonds), which red shifted to $260 \mathrm{~nm}$ for $\mathrm{rGO}$, and the absorption in the whole spectral region increases with reaction time, suggesting that $\mathrm{sp}^{2}$ carbon was restored and atoms were possibly rearranged corresponding to deoxygenation of the GO suspension under the reduction processes [18]. In addition, GO shows another broad shoulder at $290 \mathrm{~nm}$ indicating $\pi-\pi^{*}$ transitions of $\mathrm{C}=\mathrm{O}$ were observed during the entire course of GO preparation, which disappeared for rGO, suggesting that GO is reduced and the electronic conjugation within the graphene sheets is restored upon resveratrol reduction.

To investigate how $\pi$-conjugated system has been restored in the reduced form of GO, the measurement of resistivity is another important parameter. Figure 1(b) represents the changes in resistivity before and after reduction reaction of GO. It was observed that the resistance decreased substantially with the increasing reduction time. Significantly, a low sheet resistance of $\sim 1.08 \Omega$ is achieved for rGO-CuNCs, whereas $\sim 38 \Omega$ and $\sim 312 \Omega$ are measured for rGO and GO, respectively, which represents the lowest sheet resistance achieved in solution-processed rGO-CuNCs nanocomposite that is consistent with the highest degree of deoxygenation and graphitization.

Figure 2 represents the FTIR spectrum of the GO, rGO, and rGO-CuNCs. Translucent differences between GO and rGO spectrum can be observed. The presence of different type of oxygen-containing functional groups in GO was confirmed at $1382 \mathrm{~cm}^{-1}$ assigned to C-O stretching in epoxide, at $1145 \mathrm{~cm}^{-1}$ and $1650 \mathrm{~cm}^{-1}$ assigned to carboxyl, and at $3360 \mathrm{~cm}^{-1}$ assigned to $\mathrm{C}-\mathrm{OH}$ stretching. After the reduction of GO, the FTIR spectrum has a significant change. A broad peak arises at $1030 \mathrm{~cm}^{-1}$ which is assigned to alcoholic hydroxyl groups and also found a sifted peak from $1382 \mathrm{~cm}^{-1}$ (GO) to $1400 \mathrm{~cm}^{-1}$ (rGO) due to reduction of GO. Moreover, some peaks are weak and some disappeared entirely in rGO. It has been observed from rGO curve that the decreased 


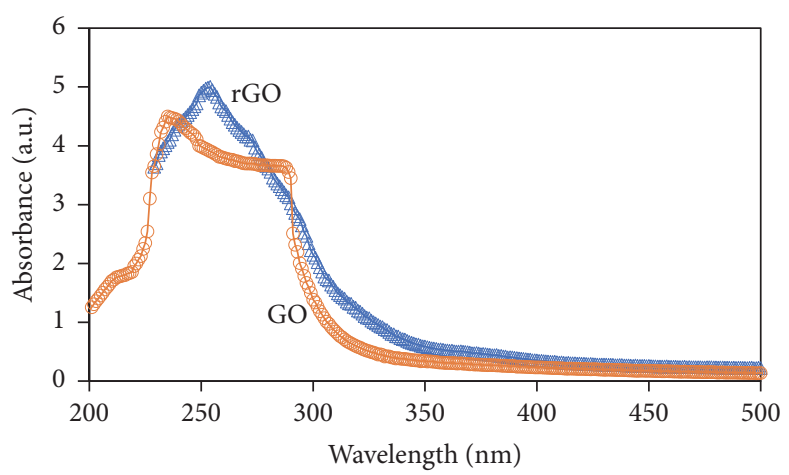

(a)

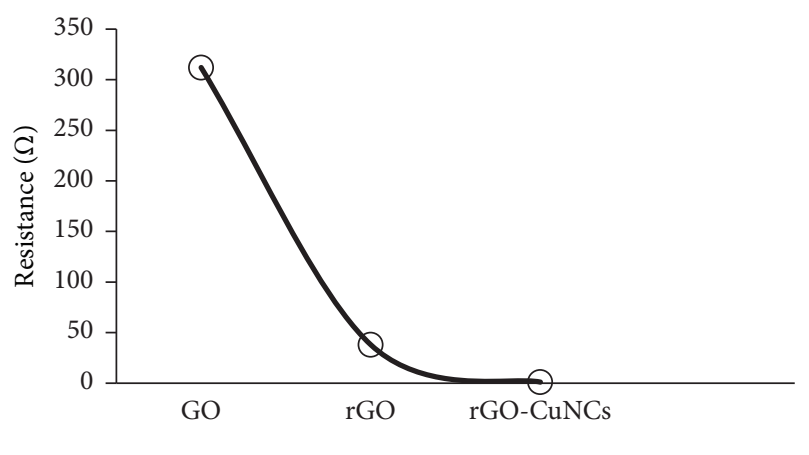

(b)

FIGURE 1: (a) UV-vis spectra of GO aqueous dispersions before (1) and after being reduced via L-AA. (b) Resistance of the as prepared GO, $\mathrm{rGO}$, and $\mathrm{rGO}-\mathrm{CuNCs}$ aqueous solutions at room temperature.

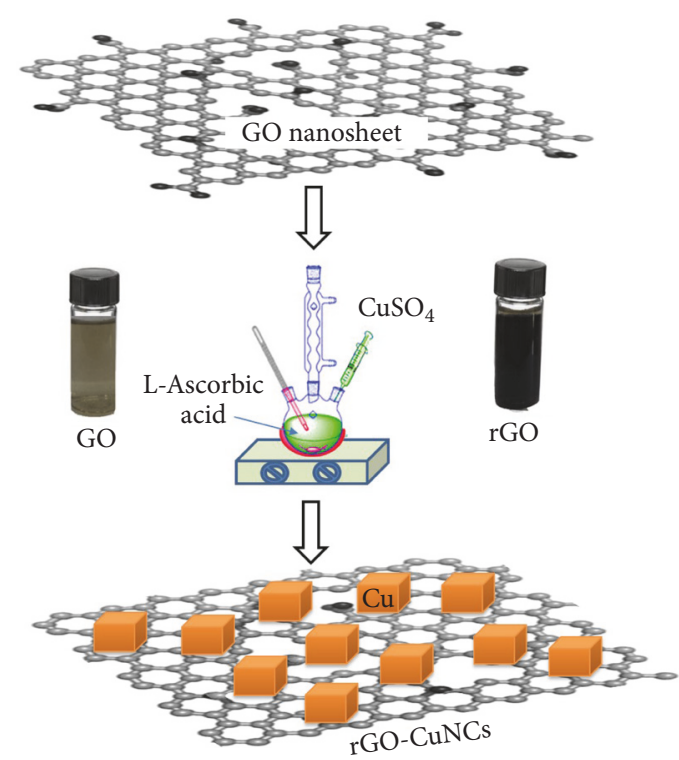

Scheme 1: Schematic representation of rGO-CuNCs preparation method.

intensity of the peak assigned for GO can be explained as mild reduction of that GO due to residual oxygen-containing functional groups [18]. After the formation of rGO-CuNPs, a translucent difference between $\mathrm{rGO}$ and rGO-CuNCs was found which indicates the formation of $\mathrm{rGO}-\mathrm{CuNCs}$. In rGOCuNCs curve, the broad peak of hydroxyl shifted to higher wave number and carboxyl groups shifted to lower wave number after the preparation of rGO-CuNCs. These could be ascribed to the synergistic effect of hydrogen bonding between CuNCs and rGO [19, 20]. Also, contrast found in FTIR spectrum at $860 \mathrm{~cm}^{-1}$ and $1400 \mathrm{~cm}^{-1}$ correspond to deformation of $\mathrm{Cu}-\mathrm{O}-\mathrm{H}$, stretching of $\mathrm{C}-\mathrm{O}$ epoxide, and other peaks before and after $\mathrm{rGO}-\mathrm{CuNCs}$ preparation which reveals that CuNCs were decorated on rGO [21]. These observations demonstrate that the molecules in rGO facilitate coordination with CuNPs species.

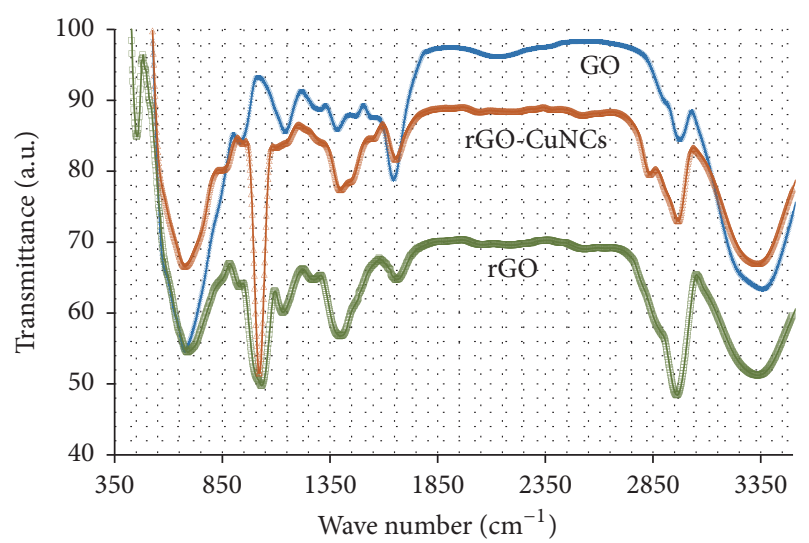

Figure 2: FTIR spectra of GO, rGO, and rGO-CuNCs.

SEM measurement was done to deduce the distribution of CuNCs onto rGO sheets and their coverage over the ITO film. The bare ITO electrode with an average surface roughness (Ra) of $1.5 \mathrm{~nm}$ was shown in Figure 3(a), whereas Figure 3(b) represents the ultraflat monolayer of rGO sheets prepared from 100 times dilute aqueous solution of supplied GO. Figure 3(b) shows the monolayer of GO sheets deposited from supplied GO solutions after successive sonication and centrifuge treatment. AFM image of the single flake shows high quality GO with well-defined edges and without surface defects in the form of cracks and wrinkles (Figure 3(d)). The average height of the GO sheets was observed in the range of 0.75-0.9 nm, which corresponds to the single layer GO sheet. The morphologies of the $\mathrm{Cu}$ nanocubes were also characterized. Figure 3(c) shows the SEM images of the $\mathrm{Cu}$ nanocubes at different magnifications as deposited on ITO electrode. It can be seen that the as-synthesized CuNCs exhibit uniform cubic shape with no other impurity particles and aggregates. To investigate the dispersion and nucleation of CuNCs on the reduced graphene nanosheets, the ITO/rGO/CuNCs films were studied, as shown in Figure 3(e). The SEM micrograph 


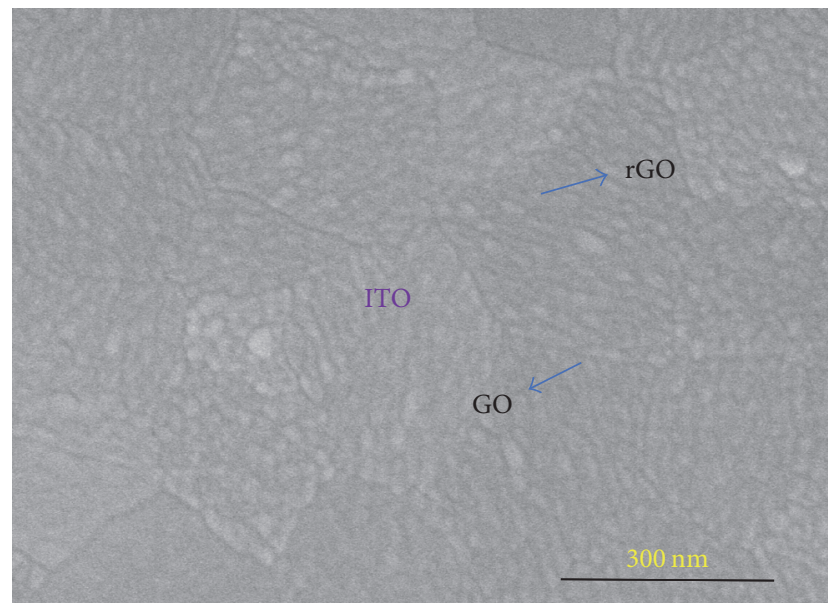

(a)

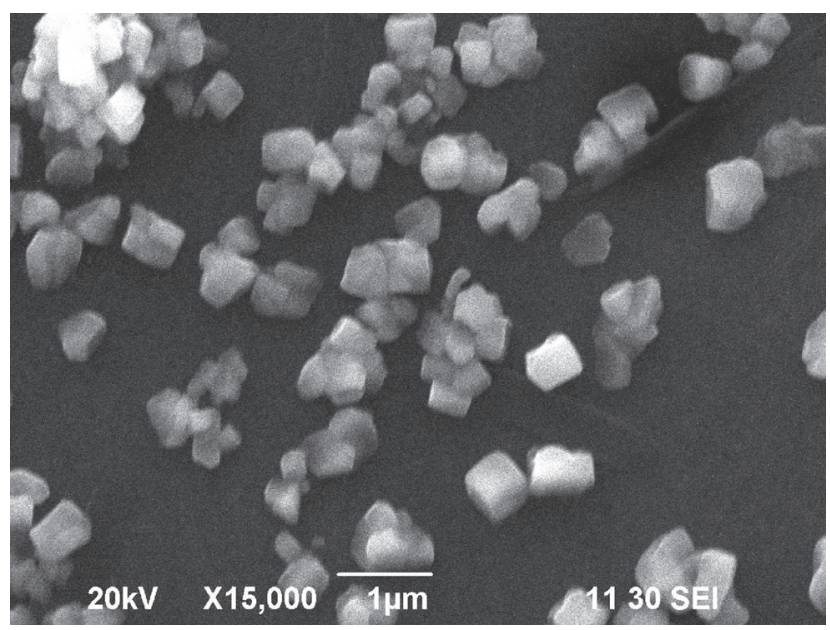

(c)

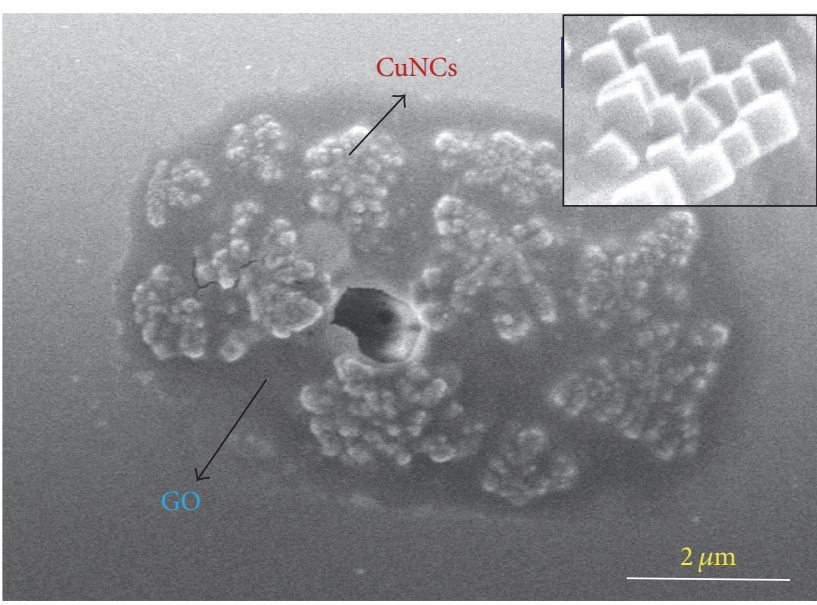

(e)

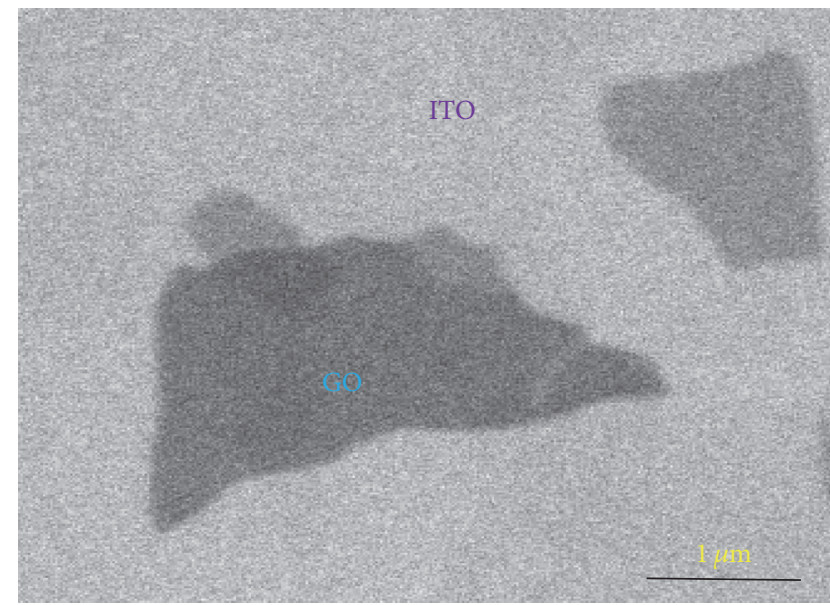

(b)

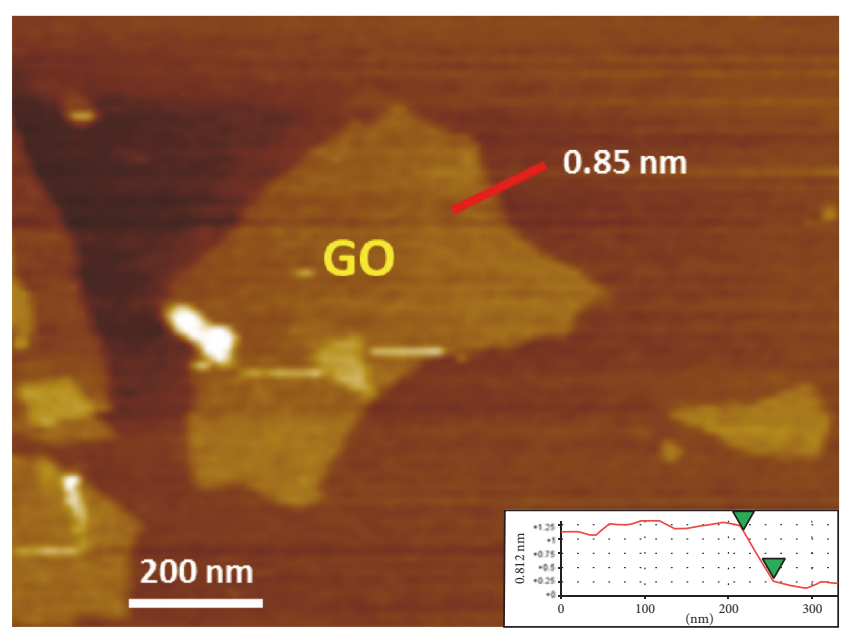

(d)

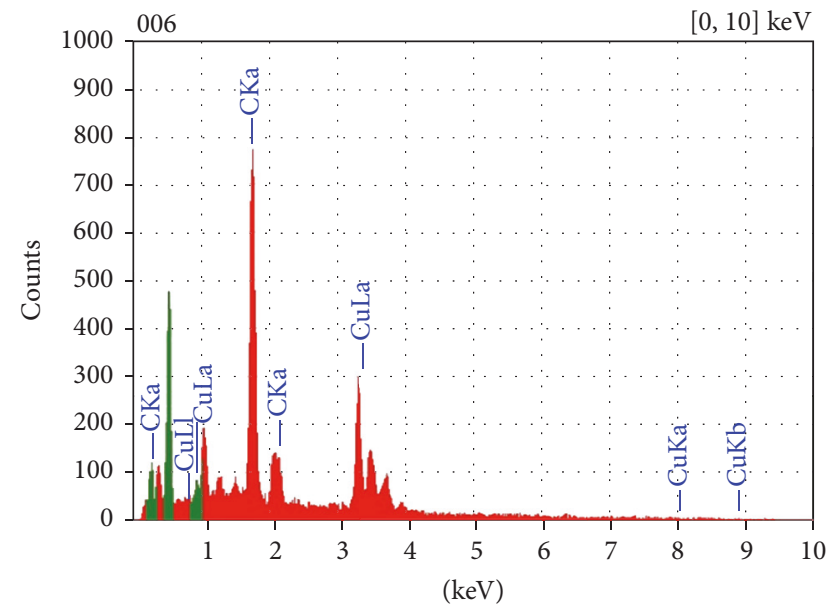

(f)

FIGURE 3: SEM images of (a) bare ITO electrode; (b) GO nanosheet on ITO; (c) CuNCs deposited on ITO; (d) noncontact mode AFM image of unreduced single GO sheets with an average height of $0.85 \mathrm{~nm}$; (e) SEM image of decorated ITO/rGO-CuNCs; (f) EDS of ITO/rGO-CuNCs film. 
at higher magnification (Figure 3(e); inset) revealed that the CuNCs were uniformly anchored on graphene sheets. By contrast, the particle size of CuNCs was much bigger as $300 \mathrm{~nm}$, which due to the aggregation of CuNCs without graphene sheets acted as nucleation sites to facilitate the formation of small crystals during the reduction process. The composition of the rGO-CuNCs is determined by energy-dispersive spectroscopy (EDS) experiment, as is supplied in Figure 3(f) that confirms that the prepared film consists of CuNCs. It was reported by many researchers that basal planes of GO and rGO nanosheets consist of negatively charged epoxy and carboxyl groups $[2,3,9-11,14]$. These functional groups can be used as anchors for the adsorption of positive metal ions (e.g., $\mathrm{Cu}^{+}$) in a solution due to the electrostatic adsorption principle. The residual functional groups or, exactly speaking, the oxygen centers on the rGO sheets act as the nucleation center for nanocubes and stabilize them after growth. This guarantees the homogeneous nucleation and uniform distribution of CuNCs on the substrate.

To elucidate the electrochemical properties of rGOCuNCs decorated sheets, we are now planning to study scanning tunneling microscopy (STM), XPS, XRD, and so forth. The details on microscopy and spectroscopy studies will be discussed in future as a full paper.

\section{Conclusion}

We have demonstrated a facile method for the synthesis of copper nanocubes decorated reduced graphene oxide (rGO-CuNCs) sheets at low temperature. The resultant rGO-CuNCs film that provides a very low resistance, welldispersed $\mathrm{Cu}$ nanocubes, and strong interaction between $\mathrm{Cu}$ nanocubes and rGO nanosheet is endowed by very simple chemical reduction method. The prepared film seems to be very useful for wide applications, specially for nanodevice fabrication and electrocatalytic activity.

\section{Conflicts of Interest}

The authors declare that they have no conflicts of interest.

\section{References}

[1] T. Jiao, H. Guo, Q. Zhang et al., "Reduced Graphene OxideBased Silver Nanoparticle-Containing Composite Hydrogel as Highly Efficient Dye Catalysts for Wastewater Treatment," Scientific Reports, vol. 5, Article ID 11873, 2015.

[2] M. Z. H. Khan, "Graphene oxide modified electrodes for dopamine sensing," Journal of Nanomaterials, vol. 2017, Article ID 8178314, 11 pages, 2017.

[3] X. Li, B. K. Tay, J. Li, D. Tan, C. W. Tan, and K. Liang, "Mildly reduced graphene oxide-Ag nanoparticle hybrid films for surface-enhanced Raman scattering," Nanoscale Research Letters, vol. 7, pp. 1-10, 2012.

[4] P. T. Yin, S. Shah, M. Chhowalla, and K.-B. Lee, "Design, synthesis, and characterization of graphene-nanoparticle hybrid materials for bioapplications," Chemical Reviews, vol. 115, no. 7, pp. 2483-2531, 2015.
[5] R. Ortega-Amaya, Y. Matsumoto, A. M. Espinoza-Rivas, M. A. Pérez-Guzmán, and M. Ortega-López, "Development of highly faceted reduced graphene oxide-coated copper oxide and copper nanoparticles on a copper foil surface," Beilstein Journal of Nanotechnology, vol. 7, pp. 1010-1017, 2016.

[6] W. Qi, P. Li, Y. Wu et al., "Facile synthesis of $\mathrm{CoFe}_{2} \mathrm{O}_{4}$ nanoparticles anchored on graphene sheets for enhanced performance of lithium ion battery," Progress in Natural Science: Materials International, vol. 26, no. 5, pp. 498-502, 2016.

[7] H. H. El-Maghrabi, E. A. Nada, F. S. Soliman, Y. M. Moustafa, and A. E. Amin, "One pot environmental friendly nanocomposite synthesis of novel $\mathrm{TiO}_{2}$-nanotubes on graphene sheets as effective photocatalyst," Egyptian Journal of Petroleum, vol. 25, no. 4, pp. 575-584, 2016.

[8] X. An, K. Li, and J. Tang, "Cu2O/reduced graphene oxide composites for the photocatalytic conversion of CO2," ChemSusChem, vol. 7, no. 4, pp. 1086-1093, 2014.

[9] C. Xu, A. Cui, Y. Xu, and X. Fu, "Graphene oxide- $-\mathrm{TiO}_{2}$ composite filtration membranes and their potential application for water purification," Carbon, vol. 62, pp. 465-471, 2013.

[10] N. Luo, X.-J. Li, K.-X. Liu, L.-M. Ye, and T.-W. Chen, "Preparation of carbon-coated copper nanoparticles by detonation decomposition of copper ion doped sol-gel explosive precursors," Journal of Nanoparticle Research, vol. 15, no. 5, article no. 1614, 2013.

[11] S. Wang, X. Huang, Y. He et al., "Synthesis, growth mechanism and thermal stability of copper nanoparticles encapsulated by multi-layer graphene," Carbon, vol. 50, no. 6, pp. 2119-2125, 2012.

[12] J. Zhai, X. Tao, Y. Pu, X.-F. Zeng, and J.-F. Chen, "Controllable synthesis and characterization of novel copper-carbon core-shell structured nanoparticles," Materials Research Bulletin, vol. 46, no. 6, pp. 865-870, 2011.

[13] Q. Wang, Q. Wang, M. Li, S. Szunerits, and R. Boukherroub, "Preparation of reduced graphene oxide/Cu nanoparticle composites through electrophoretic deposition: application for nonenzymatic glucose sensing," RSC Advances, vol. 5, no. 21, pp. 15861-15869, 2015.

[14] D.-B. Xiong, M. Cao, Q. Guo et al., "High content reduced graphene oxide reinforced copper with a bioinspired nanolaminated structure and large recoverable deformation ability," Scientific Reports, vol. 6, no. 1, 2016.

[15] H. Gao, Y. Wang, F. Xiao, C. B. Ching, and H. Duan, "Growth of copper nanocubes on graphene paper as free-standing electrodes for direct hydrazine fuel cells," Journal of Physical Chemistry C, vol. 116, no. 14, pp. 7719-7725, 2012.

[16] M. Liu, R. Liu, and W. Chen, "Graphene wrapped $\mathrm{Cu}_{2} \mathrm{O}$ nanocubes: Non-enzymatic electrochemical sensors for the detection of glucose and hydrogen peroxide with enhanced stability," Biosensors and Bioelectronics, vol. 45, no. 1, pp. 206-212, 2013.

[17] K. Morioku, N. Morimoto, Y. Takeuchi, and Y. Nishina, "Concurrent Formation of Carbon-Carbon Bonds and Functionalized Graphene by Oxidative Carbon-Hydrogen Coupling Reaction," Scientific Reports, vol. 6, Article ID 25824, 2016.

[18] J. Zhang, H. Yang, G. Shen, P. Cheng, J. Zhang, and S. Guo, "Reduction of graphene oxide vial-ascorbic acid," Chemical Communications, vol. 46, no. 7, pp. 1112-1114, 2010.

[19] X. Yang, Y. Tu, L. Li, S. Shang, and X.-M. Tao, "Well-dispersed chitosan/graphene oxide nanocomposites," ACS Applied Materials and Interfaces, vol. 2, no. 6, pp. 1707-1713, 2010. 
[20] M. N. K. Chowdhury, M. D. H. Beg, M. R. Khan, and M. F. Mina, "Synthesis of copper nanoparticles and their antimicrobial performances in natural fibres," Materials Letters, vol. 98, pp. 26-29, 2013.

[21] D. C. B. Alves, R. Silva, D. Voiry, T. Asefa, and M. Chhowalla, "Copper nanoparticles stabilized by reduced graphene oxide for $\mathrm{CO}_{2}$ reduction reaction," Materials for Renewable and Sustainable Energy, vol. 4, no. 1, 2015. 

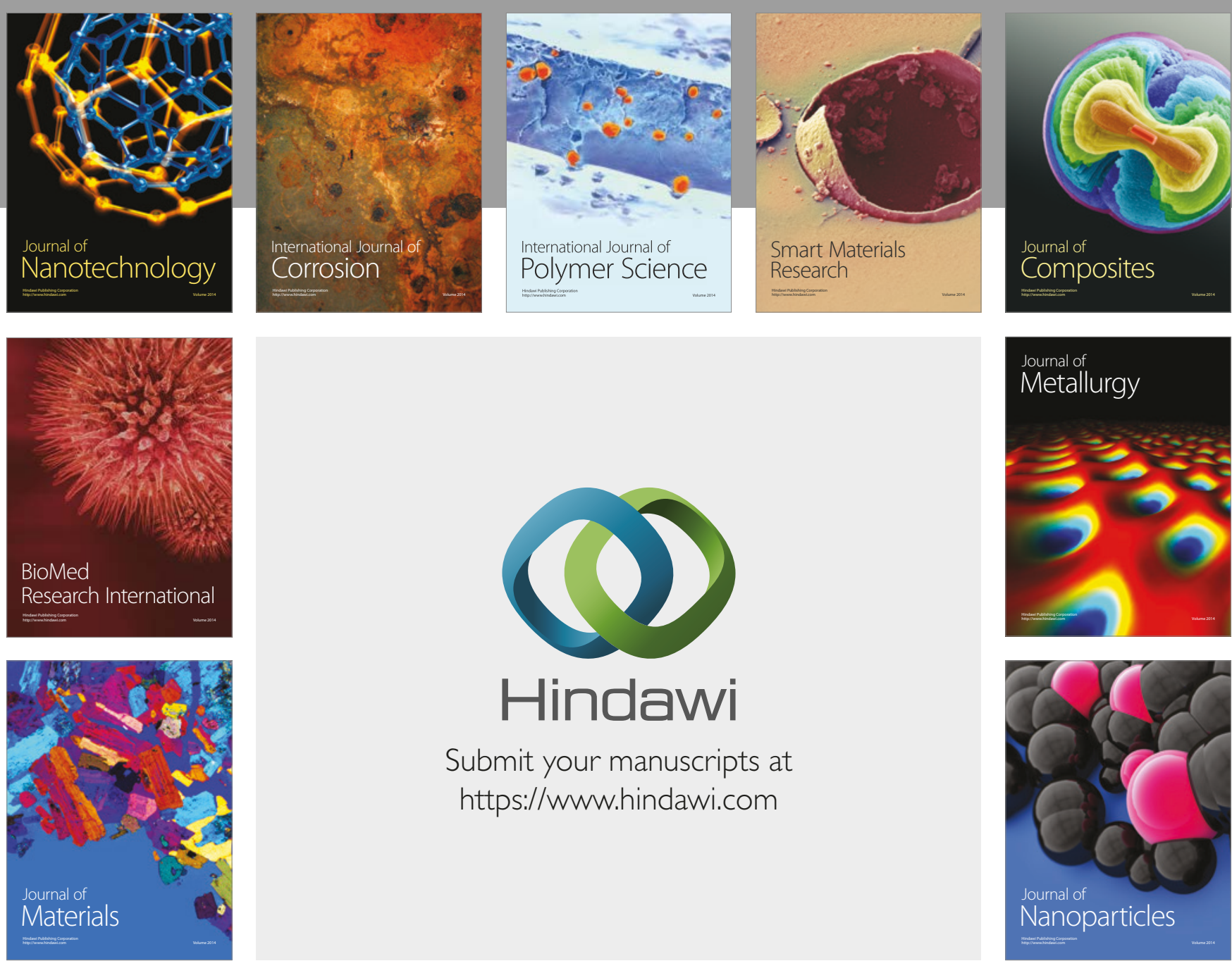

\section{Hindawi}

Submit your manuscripts at

https://www.hindawi.com
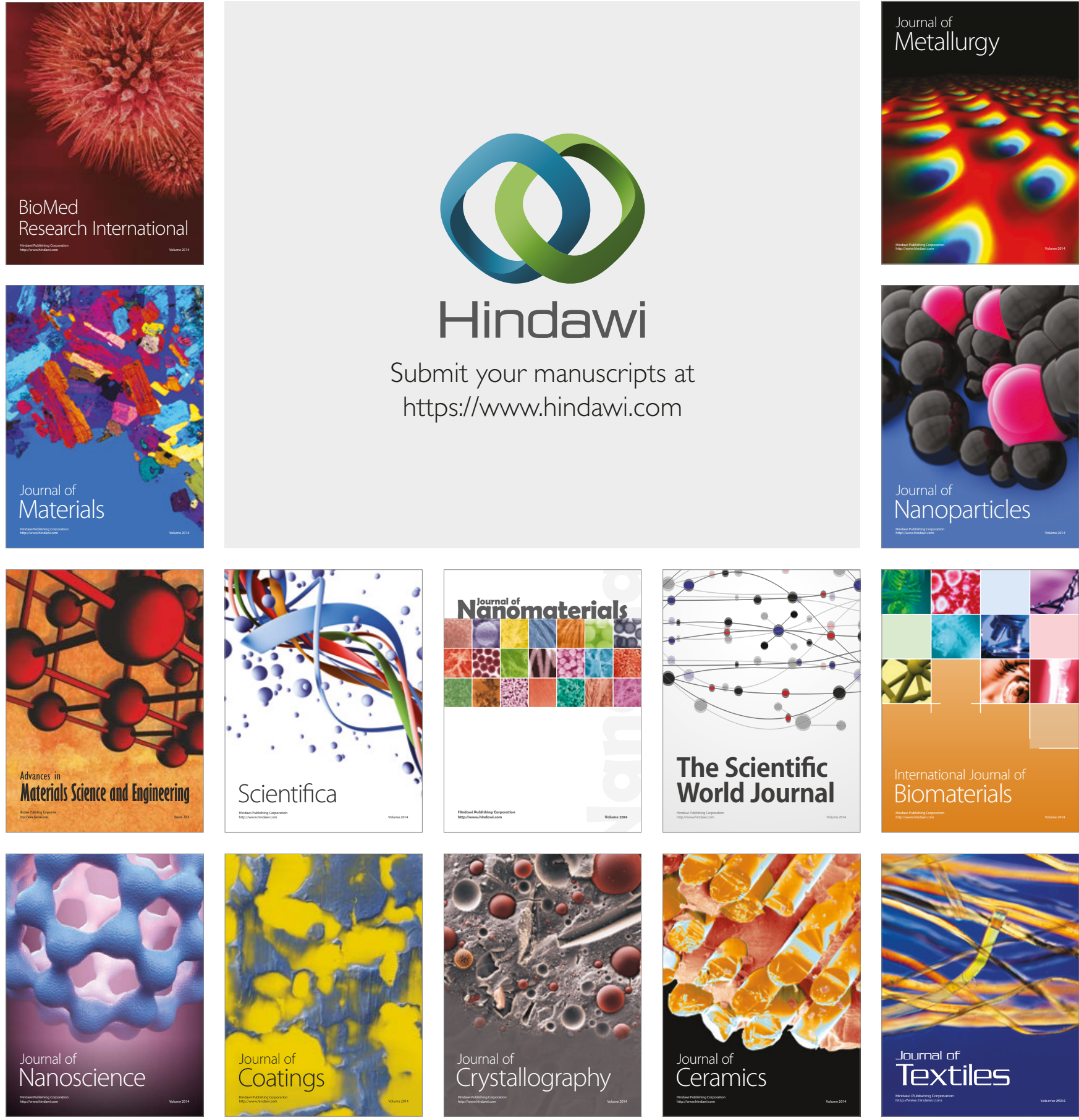

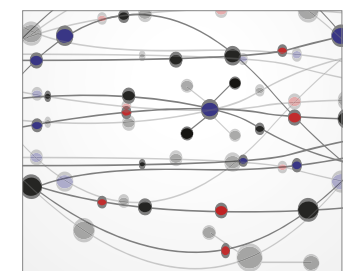

The Scientific World Journal
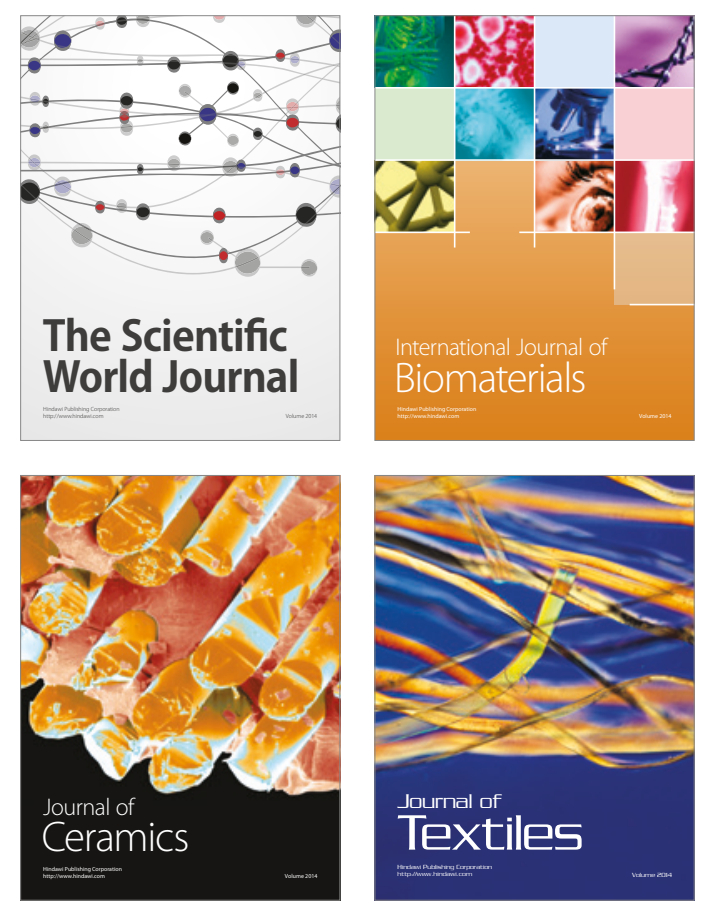